\title{
CLINICAL ARTICLES
}

\section{Dapsone/Pyrimethamine May Prevent Mycobacterial Disease in Immunosuppressed Patients Infected with the Human Immunodeficiency Virus}

\author{
Milos Opravil, Marc Pechère, Adriano Lazzarin, \\ Alison Heald, Sigmund Rüttimann, Anne Iten, \\ Hansjakob Furrer, Daniel Oertle, Gerard Praz, \\ Dominique A. Vuitton, Bernard Hirschel, \\ and Ruedi Lüthy
}

\author{
From the University Hospitals of Zurich, Geneva, Basel, Lausanne, and \\ Bern, Switzerland; Kantonsspital St. Gallen, Institut central des hôpitaux \\ valaisans, Sion, Switzerland; H. San Raffaele. Milan, Italy; and \\ University Hospital Besançon, France
}

\begin{abstract}
Dapsone exhibits activity against Mycobacterium tuberculosis and Mycobacterium avium complex (MAC) in vitro. We retrospectively examined the incidence of mycobacterial diseases within a randomized prospective trial of prophylaxis for Pneumocystis carinii pneumonia and toxoplasmosis. Of 501 participants who had not previously had a mycobacterial disease, 274 received dapsone/pyrimethamine (200/75 $\mathrm{mg}$ once weekly) and 227 received aerosolized pentamidine ( $300 \mathrm{mg}$ once every 4 weeks). The median CD4 lymphocyte count was $113 / \mu \mathrm{L}$, and the median duration of treatment was 369 days. Six cases of tuberculosis, 22 of MAC infection, and 3 of Mycobacterium genavense disease occurred during treatment. Stratified by baseline CD4 lymphocyte counts, the annual product-limit incidence of mycobacterial disease was $5 \%$ during treatment with dapsone/pyrimethamine vs. $12 \%$ during treatment with aerosolized pentamidine for patients whose counts were $0-24 / \mu \mathrm{L}, 0$ vs. $12 \%$ for those whose counts were $25-49 / \mu \mathrm{L}$, and $7 \%$ vs. $9 \%$ for those whose counts were $50-99 / \mu \mathrm{L}$. Adjusted for CD4 lymphocyte counts at start of treatment, the relative risk for patients receiving dapsone/pyrimethamine was 0.47 (95\% confidence interval, $0.19-1.16 ; P=.10$ ). This inexpensive and simple regimen may prevent mycobacterial diseases and warrants further investigation as a means of prophylaxis for multiple opportunistic diseases.
\end{abstract}

The risk of tuberculosis for human immunodeficiency virus (HIV)-infected patients is $>100$-times that for the general population [1]. In the presence of severe immune deficiency, however, nontuberculous mycobacterioses-mainly Mycobacterium avium complex (MAC) disease-become predominant [2]. In fact, the annual incidence of MAC bacteremia was as high as $20 \%$ in a study of patients with AIDS and CD4 lymphocyte counts of $20-39 / \mu \mathrm{L}$ [3]. Hence, prophylaxis for mycobacterial diseases in immunosuppressed,

Received 19 November 1993; revised 13 May 1994.

This study was presented in part at the 33 rd Interscience Conference on Antimicrobial Agents and Chemotherapy, held 17-20 October 1993 in New Orleans (abstract no. 1080).

Written informed consent was obtained from all patients, and the study was approved by the ethics committees of all participating centers.

Grant support: Swiss National AIDS Research Program (grant numbers 90-7051 and 91-7059).

Current addresses: Dr. Heald, Division of Infectious Diseases, Duke University Medical Center, Durham, North Carolina 27710; Dr. Rüttimann, Medizinische Abteilung, Kantonsspital, 8208 Schafthausen, Switzerland; and Dr. Oertle, Medizinische Abteilung, Spital Limmattal, 8952 Schlieren, Switzerland

Reprints or correspondence: Dr. M. Opravil, Division of Infectious Diseases, University Hospital, 8091 Zurich, Switzerland.

Clinical Infectious Diseases 1995;20:244-9

(C) 1995 by The University of Chicago. All rights reserved. 1058-4838/95/2002-0004\$02.00
HIV-infected patients is generating increased interest. Based on the results of two prospective studies [2], a recommendation has recently been issued that rifabutin be used as prophylaxis for MAC disease in patients with CD4 lymphocyte counts of $<100 / \mu \mathrm{L}$ [4]. Despite the proven antimycobacterial activity of numerous other agents, no controlled data exist concerning their prophylactic efficacy.

We conducted a prospective, randomized trial comparing dapsone/pyrimethamine (200/75 mg once weekly) with aerosolized pentamidine ( $300 \mathrm{mg}$ once every 4 weeks) as prophylaxis for Pneumocystis carinii pneumonia (PCP) and toxoplasmic encephalitis [5]. Dapsone is active against Mycobacterium tuberculosis and MAC in vitro [6, 7] and may have prophylactic efficacy against MAC in HIV-infected patients [8]. We therefore decided to retrospectively examine the incidence of tuberculous and nontuberculous mycobacterial diseases in participants of our study to assess the prophylactic efficacy of dapsone/pyrimethamine in comparison with that of aerosolized pentamidine.

\section{Methods}

\section{Study Population}

The participants were adults with symptomatic HIV infection and/or $<200 \mathrm{CD} 4$ lymphocytes per $\mu \mathrm{L}$. Persons were 
not disqualified if they had previously had PCP or had received prophylaxis with aerosolized pentamidine, and simultaneous antiretroviral treatment was allowed. Exclusion criteria were prior toxoplasmic encephalitis, current treatment with drugs active against $P$. carinii or Toxoplasma gondii. pregnancy, active iv drug use, and a serum creatinine level $>120 \mu \mathrm{mol} / \mathrm{L}$. An additional exclusion criterion for the present analysis was the occurrence of mycobacterial infection prior to randomization. The study design was approved by the ethics committees of the participating hospitals, and all participants gave informed consent.

\section{Study Design}

This controlled, open-label trial was conducted in 7 centers in Switzerland, 4 centers in Milan, Italy, and 1 center in Besançon, France. Participants were assigned by randomization to one of two treatment groups to receive either 200 $\mathrm{mg}$ of dapsone and $75 \mathrm{mg}$ of pyrimethamine once weekly or $300 \mathrm{mg}$ of aerosolized pentamidine (by means of a Respirgard II [Marquest, Englewood, NJ]) every 4 weeks, under the supervision of a nurse. Block randomization with a $1: 1$ ratio was used, and stratification was by center, history of previous PCP, and positivity or negativity for toxoplasmal IgG. Folinic acid was not routinely administered. The protocol allowed a change from one treatment group to the other in cases of intolerance or occurrence of breakthrough PCP. For the last $28 \%$ of participants enrolled in the study, the randomization ratio was changed to $2: 1$ in favor of the dapsone/ pyrimethamine regimen to compensate for a high rate of switches to treatment with aerosolized pentamidine.

The participants were evaluated every 4 weeks during treatment, or until the study ended on 31 December 1992 , for symptoms, opportunistic infections, compliance, adverse events, and laboratory data. Routine mycobacterial cultures were not performed for asymptomatic patients.

The present retrospective subanalysis of the trial focused on the incidence of mycobacterial diseases. Participants with known disease or colonization with $M$. tuberculosis, MAC, or other pathogenic mycobacteria prior to randomization were excluded from this analysis. The endpoints were diagnosis of tuberculous or nontuberculous mycobacterial disease, as defined by the occurrence of symptoms (such as fever, night sweats, and/or weight loss) that mandated a diagnostic workup, the detection of pathogenic mycobacteria in the respective organ(s), and-in cases of localized nontuberculous disease- the response to antimycobacterial treatment. One patient with pulmonary colonization due to MAC whose blood cultures were repeatedly negative was not considered for this analysis. The occurrence of mycobacterial diseases was evaluated in relation to treatment actually received; observation ended 30 days after the stop of the respective treatment. Patients who switched treatment groups were evaluated during the actual duration of each treatment, and CD4
Table 1. Baseline characteristics of the 501 participants in the study, who were assigned by randomization to one of two treatment groups.

\begin{tabular}{|c|c|c|}
\hline \multirow[b]{2}{*}{ Characteristic } & \multicolumn{2}{|c|}{ Treatment group } \\
\hline & $\begin{array}{c}\mathrm{DP} \\
(n=274)\end{array}$ & $\begin{array}{c}\mathrm{AP} \\
(n=227)\end{array}$ \\
\hline Age $(y)$, mean & 36 & 35 \\
\hline Male $(\%)$ & 83 & 84 \\
\hline Homosexual or bisexual (\%) & 43 & 35 \\
\hline Injection drug use $(\%)$ & 34 & 39 \\
\hline Previous CDC stage IVC 1 infection* (\%) & 27 & 27 \\
\hline $\begin{array}{l}\text { Previous use of zidovudine or } \\
\text { didanosine }(\%)\end{array}$ & 90 & 91 \\
\hline $\begin{array}{l}\text { CD4 lymphocytes per } \mu \mathrm{L} \text {. } \\
\text { median no. (range) }\end{array}$ & $117(1-610)$ & $106(1-530)$ \\
\hline
\end{tabular}

NOTE. $\quad \mathrm{DP}=$ dapsone/pyrimethamine; $\mathrm{AP}=$ aerosolized pentamidine None of the parameters differed significantly between the two treatment groups.

$*$ i.e., a previous AIDS-defining opportunistic infection $(C D C=$ Centers for Disease Control and Prevention).

lymphocyte counts at the time of the switch were considered the baseline value for the second treatment.

\section{Statistical Analysis}

Baseline characteristics were compared by means of the $\chi^{2}$ test or the Mann-Whitney test, as appropriate. Intraindividual changes in CD4 lymphocyte counts were compared by the Wilcoxon signed-rank test. The probability of remaining free of mycobacterial disease was calculated by means of the Kaplan-Meier life-table analysis. Multivariate Cox (proportional hazards) regression analysis was used to estimate factors predictive of mycobacterial disease; the relative risk in patients treated with dapsone/pyrimethamine was adjusted for CD4 lymphocyte counts at start of treatment. A $P$ value of $<.05$ was considered statistically significant.

\section{Results}

\section{Patients' Characteristics}

A total of 533 patients were randomly assigned to treatment. Thirty-two had a previously diagnosed mycobacterial disease and were therefore excluded from the present analysis; nine had nontuberculous mycobacteriosis, and 23 had tuberculosis. The remaining 501 participants were not receiving any drugs with antimycobacterial activity at the time of randomization and could therefore be evaluated for occurrence of mycobacterial disease during the study. Of these 501 patients, 274 were assigned to the dapsone/pyrimethamine treatment group and 227 to the aerosolized pentamidine treatment group.

The baseline characteristics of the two groups were well balanced (table 1); the average age was 35.5 years, $83 \%$ were 
Table 2. Mycobacterial diseases that occurred during the study in 31 of the participants.

\begin{tabular}{lllr}
\hline Disease & \multicolumn{1}{c}{ Site of detection } & $\begin{array}{c}\text { Mycobacterium } \\
\text { species implicated }\end{array}$ & $\begin{array}{c}\text { No. of participants } \\
\text { infected }\end{array}$ \\
\hline Systemic & Blood, bone marrow & MAC & 21 \\
& & M. genavense & 2 \\
& & M. tuberculosis & 2 \\
Localized & M. africanum & $1^{*}$ \\
Pneumonia & Sputum, bronchoalveolar & M. tuberculosis & 2 \\
Meningitis & lavage fluid & MAC & 1 \\
Lymph node abscess & CSF & M. tuberculosis & 1 \\
\hline
\end{tabular}

* This was counted as one of the six cases of tuberculosis.

male, and the main risk factors for HIV infection were homosexuality (39\%) and injection drug use (37\%). For the entire population, the median CD4 lymphocyte count was $113 / \mu \mathrm{L}$. Only $27 \%$ had had an AIDS-defining opportunistic infection, but $90 \%$ were receiving antiretroviral treatment at the time of randomization.

The median duration of study medication was 369 days, corresponding to 504.5 patient-years of treatment (199.4 for dapsone/pyrimethamine and 305.1 for aerosolized pentamidine). Ninety-nine patients switched from the originally assigned treatment group to the other one: 83 because of intolerance for dapsone/pyrimethamine, 7 because of intolerance for aerosolized pentamidine, 6 because of breakthrough PCP ( 2 were receiving dapsone/pyrimethamine and 4 were receiving aerosolized pentamidine), and 3 for other reasons (e.g., possible but not proven PCP and poor compliance). The CD4 lymphocyte counts of the 88 patients switching from dapsone/pyrimethamine to aerosolized pentamidine were significantly lower at the time of the switch (median, $57.5 / \mu \mathrm{L} ; P<.005)$ than at the time of randomization; the incidence of mycobacterial diseases was therefore adjusted on the basis of CD4 lymphocyte counts determined at the start of each treatment.

\section{Mycobacterial Diseases}

A total of 31 cases of mycobacterial disease were diagnosed during treatment: 6 of tuberculosis (involving 1 patient receiving dapsone/pyrimethamine and 5 receiving aerosolized pentamidine), 22 of MAC disease (5, dapsone/ pyrimethamine; 17 , aerosolized pentamidine), and 3 of $M$. genavense infection (all, aerosolized pentamidine). All patients were symptomatic; infection was detected by means of blood or bone marrow cultures and was therefore disseminated in $26(84 \%)$ of the 31 patients (table 2). Among the 25 patients with MAC or $M$. genavense infection, all had fever, $72 \%$ had weight loss, $56 \%$ had night sweats, and $36 \%$ had diarrhea. Sixteen received antimycobacterial treatment for at least 1 month, and $14(88 \%)$ of them-including the two with localized disease (pneumonia and lymph node abscess)-responded favorably with respect to their clinical symptoms.

Eleven patients had a mycobacterial disease after they switched from dapsone/pyrimethamine to aerosolized pentamidine (median number of days after switch, 211; range, 38-464 days) (table 3). Among the 31 patients with mycobacterial disease, 18 (58\%) had baseline CD4 lymphocyte counts $<100 / \mu \mathrm{L}$. Among those with such counts, the incidence of mycobacterial disease was 3.1 per 100 patient-years (95\% confidence interval [CI], 0.3-8.8) during treatment with dapsone/pyrimethamine vs. $11.4(\mathrm{CI}, 6.5-11.7)$ during treatment with aerosolized pentamidine.

In a multivariate Cox (proportional hazards) regression analysis, patients receiving dapsone/pyrimethamine had a relative risk of $0.47(95 \% \mathrm{CI}, 0.19-1.16 ; P=.10)$ of developing mycobacterial disease (risk was adjusted for CD4 lymphocyte counts at the start of treatment). In an analysis considering only patients with CD4 lymphocyte counts $<100 / \mu \mathrm{L}$, the adjusted relative risk was $0.30(95 \% \mathrm{CI}, 0.07-$ $1.32 ; P=.11$ ). Because the development of mycobacterial disease was independently predicted by the CD4 lymphocyte counts at the start of treatment $(P=.021)$, we stratified the patients' baseline counts into the ranges of 0-24, 25-49, $50-99,100-199$, and $>200 / \mu \mathrm{L}$ and calculated the product limit incidence of mycobacterial disease within these ranges at different observation times. In all strata with CD4 lymphocyte counts $<200 / \mu \mathrm{L}$, mycobacterial diseases developed earlier and more frequently in patients receiving aerosolized pentamidine than in those receiving dapsone/ pyrimethamine (nonsignificant trend; table 4).

\section{Mortality}

Seventy-five patients died during treatment or within 30 days of its discontinuation; 27 were receiving dapsone/ pyrimethamine and 48 were receiving aerosolized pentamidine at the time of death. The rate of death per 100 patient-years was similar: 13.5 for patients receiving dap- 
Table 3. The duration of treatment and the clinical characteristics and events during such treatment for the 501 participants.

\begin{tabular}{|c|c|c|c|c|}
\hline \multirow[b]{2}{*}{ Variable } & \multicolumn{2}{|c|}{ Assigned treatment } & \multicolumn{2}{|c|}{$\begin{array}{l}\text { Treatment } \\
\text { switched to }\end{array}$} \\
\hline & DP & AP & DP & AP \\
\hline No. of patients & 274 & 227 & 11 & 88 \\
\hline Median duration of treatment (person-years) & 191.1 & 229.1 & 8.3 & 76.0 \\
\hline Median no. of CD4 lymphocytes per $\mu \mathrm{L}$ & 117 & 106 & $119^{*}$ & $57.5^{*}$ \\
\hline Development of tuberculosis (no. of patients) ${ }^{\dagger}$ & 1 & 2 & 0 & 3 \\
\hline \multicolumn{5}{|l|}{ Development of NTM, due to MAC or } \\
\hline M. genavense (no. of patients) $)^{\dagger}$ & 3 & 12 & 2 & 8 \\
\hline No. of deaths ${ }^{\dagger}$ & 24 & 32 & 3 & 16 \\
\hline
\end{tabular}

NOTE. $\quad \mathrm{DP}=$ dapsone/pyrimethamine: $\mathrm{AP}=$ aerosolized pentamidine; $\mathrm{NTM}=$ non-tuberculous $\mathrm{myco}-$ bacteriosis.

* At the time of switch.

${ }^{\dagger}$ During the treatment or within 30 days after that treatment ended.

sone/pyrimethamine and $\mathbf{1 5 . 7}$ for patients receiving aerosolized pentamidine.

\section{Discussion}

The subanalysis of this randomized prophylactic trial shows a trend toward decreased incidence of mycobacterial diseases in patients receiving dapsone/pyrimethamine vs. aerosolized pentamidine. The prophylactic effect was more pronounced in patients whose CD4 lymphocyte counts were $<100 / \mu \mathrm{L}$ at the start of treatment. Among the mycobacterial diseases observed during the study, disseminated MAC infection was most frequent, followed by pulmonary and extrapulmonary tuberculosis and disease due to $M$. genavense. All mycobacterial diseases diagnosed involved patients who were symptomatic and required an appropriate diagnostic workup; therefore, the observed reduction in incidence concerned clinically manifested mycobacterial diseases and had a direct impact on the rate of morbidity among recipients of dapsone/pyrimethamine. These results support the hypothesis that dapsone/pyrimethamine might have prophylactic efficacy against mycobacteria; however, the results must be interpreted with caution, as this study was a retrospective subanalysis of a prospective trial originally designed to assess prevention of PCP and toxoplasmosis rather than antimycobacterial efficacy.

Dapsone exhibits in vitro activity against both $M$. tuberculosis and MAC [6, 7], has moderate antituberculous activity in vivo $[9,10]$, and has been used as part of a combination regimen to treat tuberculosis [11]. Although dapsone did not show therapeutic efficacy against murine MAC infection, prophylactic administration of dapsone was effective in reducing MAC bacteremia and the number of bacteria in different organs in the same model [12]. In a recent retrospective analysis, dapsone also tended to lower the incidence of MAC bacteremia in HIV-infected patients [8]. Considering

Table 4. Product limit incidence of mycobacterial disease, stratified by patients' CD4 lymphocyte counts at the time treatment started.

\begin{tabular}{|c|c|c|c|c|c|c|}
\hline \multirow{2}{*}{$\begin{array}{l}\text { Treatment } \\
\text { received }\end{array}$} & \multirow{2}{*}{$\begin{array}{c}\text { CD4 } \\
\text { lymphocytes } / \mu \mathrm{L}\end{array}$} & \multirow{2}{*}{$\begin{array}{c}\text { No. of patients } \\
\text { observed }\end{array}$} & \multicolumn{4}{|c|}{ Incidence $(\% \pm S D)$} \\
\hline & & & $3 \mathrm{mo}$ & $6 \mathrm{mo}$ & 9 mo & $12 \mathrm{mo}$ \\
\hline \multirow[t]{5}{*}{ DP } & $0-24$ & 43 & 0 & $5 \pm 4$ & $5 \pm 4$ & $5 \pm 4$ \\
\hline & $25-49$ & 35 & 0 & 0 & 0 & 0 \\
\hline & $50-99$ & 48 & 0 & 0 & 0 & $7 \pm 7$ \\
\hline & $100-199$ & 94 & 0 & 0 & 0 & $5 \pm 3$ \\
\hline & $>200$ & 65 & 0 & 0 & 0 & 0 \\
\hline \multirow[t]{5}{*}{$\mathrm{AP}$} & $0-24$ & 61 & $3 \pm 2$ & $3 \pm 2$ & $9 \pm 4$ & $12 \pm 5$ \\
\hline & $25-49$ & 42 & 0 & $3 \pm 3$ & $12 \pm 6$ & $12 \pm 6$ \\
\hline & $50-99$ & 58 & 0 & $4 \pm 3$ & $7 \pm 4$ & $9 \pm 5$ \\
\hline & $100-199$ & 102 & 0 & $1 \pm 1$ & $3 \pm 2$ & $6 \pm 3$ \\
\hline & $>200$ & 52 & 0 & 0 & 0 & 0 \\
\hline
\end{tabular}

NOTE. $\quad \mathrm{DP}=$ dapsone/pyrimethamine; $\mathrm{AP}=$ aerosolized pentamidine. 
the presence of dihydrofolate reductase in mycobacteria [13, 14] and its possible inhibition by pyrimethamine, we believe the combination of dapsone and pyrimethamine theoretically may have an additive activity against mycobacteria, but we are not aware of any data reporting the effects of pyrimethamine on mycobacteria.

The estimated annual incidence of mycobacterial diseases among patients in our study who were receiving aerosolized pentamidine and whose baseline CD4 lymphocyte counts were $<50 / \mu \mathrm{L}$ was $12 \%$. This is lower than previously reported data from the United States concerning the incidence of MAC bacteremia, which indicated the rate was as high as $20 \%$ for patients with AIDS and 20-39 CD4 lymphocytes per $\mu \mathrm{L}$ and even higher with decreasing CD4 lymphocyte counts $[3,15]$. We did not perform routine blood cultures for all our patients; the diagnostic workup was mandated by symptoms and signs. The true rate of mycobacterial bacteremia therefore may have been underdiagnosed, as indicated by a study of asymptomatic patients with $<100$ CD4 lymphocytes per $\mu \mathrm{L}$ in whom routine screening detected previously unsuspected MAC bacteremia at a rate of 5.9\% [16]. A small percentage of cases of asymptomatic MAC bacteremia, however, probably would have affected both treatment groups in our study equally. The evaluation of the occurrence of mycobacterial disease is nonetheless clinically important, as the ultimate goal of prophylaxis is reduction of morbidity rather than reduction of bacteremia.

Rifabutin and clarithromycin have been previously shown to have efficacy in primary prophylaxis for MAC bacteremia in clinical studies $[2,8]$. The combination of clarithromycin and dapsone prevented experimental murine MAC infection [12] and may also be effective in humans. Until now, only rifabutin had been evaluated in a controlled trial and reduced the incidence of MAC bacteremia by $45 \%$ in comparison with the incidence in placebo recipients [2]. However, the cost of these regimens is considerable, and the cost-benefit ratio is unknown. Prophylaxis with rifabutin ( $300 \mathrm{mg}$ once daily) costs close to $\$ 200$ per month [4]. On the basis of the local incidence of mycobacterial diseases in patients with $<100 \mathrm{CD} 4$ lymphocytes per $\mu \mathrm{L}$, the prevention of one case of nontuberculous mycobacteriosis in Switzerland would cost $>\$ 66,000$ per year [17]. With a monthly cost of $\sim \$ 2$ [18], dapsone/ pyrimethamine compares favorably with other prophylactic agents. In addition, dapsone/pyrimethamine is an attractive alternative because of its potential for once-weekly administration as well as the fact that it is not used for treatment of mycobacterioses, so a possible induction of resistance would not have immediate therapeutic implications.

However, tolerance for dapsone/pyrimethamine in our study was poor; $30 \%$ of participants had to switch to aerosolized pentamidine because of intolerance. The most frequent reasons for discontinuation of the treatment were nausea, skin rash, headache, fever, and anemia [5]. The course of the hypersensitivity reactions was reversible with symptomatic treatment; no cases of sulfone syndrome $[19,20]$ or other severe cutaneous reactions were observed.

Our results confirm the significantly increased risk for the development of mycobacterial diseases in patients whose CD4 lymphocyte counts are low and-without regard to the aspect of cost-support the assessment that primary prophylaxis for MAC disease would be clinically justified for HIVinfected patients whose CD4 lymphocyte counts are $<100$ / $\mu \mathrm{L}$ [4]. Dapsone/pyrimethamine and possibly dapsone alone have already been shown to prevent toxoplasmic encephalitis in addition to being efficacious as prophylaxis for PCP [5, $21,22]$. Because a trend toward decreased incidence of mycobacterial diseases was observed in the present analysis, combined prophylaxis for multiple opportunistic diseases with dapsone/pyrimethamine seems feasible. Future studies will have to address the issues of efficacy, tolerability, availability, and cost-benefit ratio to determine the best prophylactic regimen for immunodeficient, HIV-infected patients.

\section{Acknowledgments}

The authors are indebted to all participants in the trial, to Karola Hodapp for data management, to all physicians who took care of the patients, and to the investigators at the participating Italian centers (all in Milan): H. San Raffaele (Dr. Adriano Lazzarin); Clinica Malattie Infettive, Ospedale L. Sacco (Dr. Franco Mainini); Ia Div. Malattie Infettive, Ospedale L. Sacco (Dr. Maria C. Vivirito); Centro Bianchi Bonomi IRCCS Policlinico (Dr. Alessandro Gringeri); Centro Trasfusionale, Ospedale di Bollate (Dr. Claudio Velati); and Clinica Dermatologica IRCCS Policlinico (Dr. Roberto Zerboni).

\section{References}

1. Centers for Disease Control and Prevention. Management of persons exposed to multidrug-resistant tuberculosis. MMWR Morb Mortal Wkly Rep 1992;41(RR-1 I):6 I-71.

2. Nightingale SD, Cameron DW, Gordin FM, et al. Two controlled trials of rifabutin prophylaxis against Mycobacterium avium complex infection in AIDS. N Engl J Med 1993; 329:828-33.

3. Nightingale SD, Byrd LT, Southern PM, Jockusch JD, Cal SX, Wynne BA. Incidence of Mycobacterium avium-intracellulare complex bacteremia in human immunodeficiency virus-positive patients. J Infect Dis 1992; 165 : 1082-5.

4. Masur H, Public Health Service Task Force on Prophylaxis and Therapy for Mycobacterium avium Complex. Recommendations on prophylaxis and therapy for disseminated Mrcobacterium avium complex disease in patients infected with the human immunodeficiency virus. N Engl J Med 1993;329:898-904.

5. Opravil M, Hirschel B. Lazzarin A. et al. Once weekly administration of dapsone-pyrimethamine vs. aerosolized pentamidine as combined prophylaxis for Pheumocistis carinii pneumonia and toxoplasmic encephalitis in human immunodeficiency virus-infected patients. Clin Infect Dis 1995:20:531-41.

6. Kerkering TM, Espinel-Ingroff A, Dalton H, Warren N. In-vitro susceptibility of dapsone against $M$. tuberculosis, and strains of $M$. avium, and $M$. intracellulare isolated from AIDS patients [abstract no W.A. 1024]. In: Program and abstracts of the 7th International Con- 
ference on AIDS (Florence). Rome: Istituto Superiore di Sanità, 1991.

7. Gonzalez AH, Berlin OG. Bruckner DA. In-vitro activity of dapsone and two potentiators against Mycobacterium avium complex. J Antimicrob Chemother 1989;24:19-22.

8. Hewitt RG, Maliszewski M, Goldberg M, Harmon B. Prevention of $M$. avium complex bacteremia in patients with CD4 $<200$ by rifabutin, clarithromycin or dapsone [abstract no PO-B07-1184]. In: Program and abstracts of the 9th International Conference on AIDS/IV STD World Congress (Berlin). Berlin: 1993.

9. Winkelmann E, Raether W, Wagner WH. Chemotherapeutically active nitro compounds: 2nd communication. Nitrodiphenyl sulfones. Arzneimittelforschung 1976;26: 1543-7.

10. Bartmann K. Do existing preclinical studies with dapsone support its use in short-term chemotherapy of tuberculosis in economically developed countries? Prax Klin Pneumol 1987;41:79-87.

11. Gonzalez Montaner LF, Garay C, Abbate EH, Albareda MA. A new therapeutic approach to open pulmonary tuberculosis with a combination of isoniazid, prothionamid and dapson. Prax Klin Pneumol 1978; 32:488-90.

12. Bermudez LE, Kolonoski P, Wu M, Petrofsky M, Young LS. Combination of clarithromycin (CLA) and dapsone (DDS) for prophylaxis and therapy of murine Mycobacterium avium complex (MAC) infection [abstract no 287]. In: Program and abstracts of the 33rd Interscience Conference on Antimicrobial Agents and Chemotherapy (New Orleans). Washington. DC: American Society for Microbiology, 1993.

13. Sirawaraporn W, Sirawaraporn R, Chanpongsri A, Jacobs WR Jr, Santi DV. Purification and characterization of dihydrofolate reductase from wild-type and trimethoprim-resistant Mycobacterium smegnatis. Exp Parasitol 1991;72:184-90.

14. Al Rubeai M. Dale JW. Purification and characterization of dihydrofo- late reductase from Mvcobacterium phlei. Biochem J 1986;235: 301-3.

15. Gordin F, Cohn D, Sullam P, et al. The occurrence of Mvcobacterium avium complex (MAC) bacteremia in a standardized cohort of AIDS patients [abstract no 1405]. In: Program and abstracts of the 33rd Interscience Conference on Antimicrobial Agents and Chemotherapy (New Orleans). Washington, DC: American Society for Microbiology, 1993.

16. MacGregor R. Graziani A. Prevalence of unsuspected opportunistic infections (OIs) in HIV-positive patients with CD4 counts below $100 / \mu \mathrm{L}$ [abstract no 1409]. In: Program and abstracts of the 33rd Interscience Conference on Antimicrobial Agents and Chemotherapy (New Orleans). Washington, DC: American Society for Microbiology, 1993.

17. Opravil M, Hächler I, Lüthy R. Incidence and prophylaxis of non-tuberculous mycobacteriosis in HIV-infected patients [letter]. AIDS 1994; 8:563-4.

18. Masur $\mathrm{H}$. Prevention and treatment of pneumocystis pneumonia. $\mathrm{N}$ Engl J Med 1992;327:1853-60.

19. Mohle-Boetani J, Akula SK, Holodniy M, Katzenstein D, Garcia G. The sulfone syndrome in a patient receiving dapsone prophylaxis for Pneumoc ystis carinii pneumonia. West J Med 1992: 156:303-6.

20. Grayson ML. Yung AP. Doherty RR. Severe dapsone syndrome due to weekly maloprim [letter]. Lancet 1988; 1:531.

21. Girard PM, Landman R, Gaudebout C. et al. Dapsone-pyrimethamine compared with aerosolized pentamidine as primary prophylaxis against Pneumoc!stis carinii pneumonia and toxoplasmosis in HIV infection. N Engl J Med 1993; 328:1514-20.

22. Torres RA, Barr M. Thorn M, et al. Randomized trial of dapsone and aerosolized pentamidine for the prophylaxis of Pneumocystis carinii pneumonia and toxoplasmic encephalitis. Am J Med 1993:95:57383. 